\title{
Evaluation of the long-term stability of micro-screws under different loading protocols: a systematic review
}

\author{
Jia-Nan ZHANG(a) iD \\ Hai-Ping LU(a) \\ Xi-Chen BAO(a) iD \\ Yuan $\mathrm{SHI}^{(a)}$ iD \\ Meng-Han ZHANG(a) \\ (a)Zhejiang Chinese Medical University, \\ Department of Orthodontics, College \\ of Stomatology, Hangzhou, Zhejiang \\ Province, China.
}

Declaration of Interests: The authors certify that they have no commercial or associative interest that represents a conflict of interest in connection with the manuscript.

Corresponding Author:

Hai-Ping Lu

E-mail:2639337804@qq.com

hitps://doi.org/10.1590/1807-3107bor-2019.vol33.0046

Submitted: September 25, 2018

Accepted for publication: April 8, 2019

Last revision: April 26, 2019

\begin{abstract}
The aim of this systematic review was to investigate the association between the different factors of loading protocols and the long-term stability of micro-screws from biomechanical and histological viewpoints. Searches were performed on PubMed, Embase, Cochrane Library, Wanfang and CNKI databases for animal experiments comparing loading protocols and the long-term stability of micro-screws. Among 1011 detected papers, 16 studies met the eligibility criteria and were selected for analysis. Most studies showed medium methodological quality for evaluation of micro-screws' long-term stability. Five studies reported that loading would not destroy the long-term stability of micro-screws. Three studies indicated that low-intensity immediate loading or a 3-week minimal healing time was acceptable. Two studies reported that the loading magnitude was a controversial issue with regard to the micro-screws' long-term stability. Two studies suggested that counterclockwise loading could decrease the long-term stability of micro-screws. In conclusion, immediate loading below $100 \mathrm{~g}$ force, healing time greater than 3 weeks, regular loading below $200 \mathrm{~g}$ force and a clockwise direction of force supported the long-term stability of micro-screws. Further studies relating to the combination of varying loading conditions will be needed.
\end{abstract}

Keywords: Bone Screws; Workload Stability; Animal Experimentation.

\section{Introduction}

In recent years, micro-screws have been widely used to reinforce anchorage during orthodontic therapy. Compared with traditional anchorage, such as extraoral headgear, TPA (transpalatal arch) or oral implant, micro-screws possess the advantages of small size, simple surgical insertion procedure, straightforward removal, lower cost and independence from patient compliance. ${ }^{1,2}$ However, orthodontic micro-screws tend to suffer a failure rate of about $10 \%$ to $30 \%$, which is much higher than conventional implants. ${ }^{3}$

The surrounding bone of micro-screws after insertion needs time to heal in order to provide stable support. ${ }^{4}$ However, immediate or early activation of micro-screws is proposed in order to diminish the rehabilitation time and the period of orthodontic treatment. ${ }^{5,6}$ Therefore, scholars have evaluated the impact of healing time, loading magnitude, 
and loading direction on the stability of micro-screws in animal experiments.

The stability of micro-screws includes primary stability and long-term stability. Primary stability represents the mechanical interlock of micro-screws, which depends on the amount and thickness of cortical bone surrounding the micro-screw's threads. Long-term stability means biological ability to resist drop, and this stability is related to osseointegration. Immediate or early loading may inhibit the osseointegration process between the bone and micro-screw and may cause micromovements of micro-screws. ${ }^{7}$ Many relative studies have been conducted to figure out whether micro-screws can be loaded immediately or within a few weeks after insertion and which loading protocol is more suitable for micro-screws' long-term application. However, the influence of loading protocols varies considerably in animal study results, and this causes confusion in clinical application. ${ }^{8,9}$ Therefore, analyzing and investigating the appropriate loading protocol of micro-screws is of great significance.

In clinical practice, the orthodontists' concern is which loading protocol would be more suitable. Although micro-screws are now widely used clinically, biomechanical and histological research about the influence of loading protocols on the longterm stability of micro-screws is still confusing and limited. In this study, we aimed to systematically review and critically analyze the factors of loading protocols that affect the long-term stability of orthodontic micro-screws from biomechanical and histological viewpoints.

\section{Methodology}

The protocol of this review was developed before the start of the study, and the whole process was under the guidelines of the Preferred Reporting Items for Systematic Reviews and Meta-Analyses (PRISMA) statement and the Cochrane Handbook for Systematic Reviews of Interventions (version 5.1.0). ${ }^{10}$ Because the studies in this review were based on animals, the protocol was not registered in the Prospero database.

\section{Search strategy}

An open-ended electronic search was conducted through PubMed, Embase, Cochrane Library, Wanfang and CNKI (China National Knowledge Infrastructure) databases through the end of May 2018. No setting and language restrictions were applied. The search strategy included appropriate changes in the keywords and followed the syntax rules of each database. The aim of this search was to identify all the papers dealing with the long-term stability of orthodontic micro-screws evaluated under different loading conditions and that considered biomechanical and histological parameters. Terms used in the search included micro-screw (mini-screw, micro-implant and mini-implant), load/force and stability.

A search of the selected papers' references was also performed manually. Furthermore, the American Journal of Orthodontics and Dentofacial Orthopedics, Angle Orthodontist, European Journal of Orthodontics and Clinical Oral Implants Research were manually searched.

\section{Selection criteria}

The specific inclusion criteria for studies were as follows:

a. Controlled trials in animals;

b. Studies evaluating the long-term stability of micro-screws by considering the biomechanical values of maximum removal torque or histomorphometric values of bone to implant contact;

c. The experimental group and control group defined the application of loading force.

The exclusion criteria for studies were as follows:

a. Reviews articles, case reports or clinical trials;

b. Studies exploring measurement methods.

\section{Study selection}

Studies were firstly selected by two reviewers on the basis of the title and abstract. Whenever there was a doubt about whether the study should be included, a complete article review was conducted. If there was a conflict or disagreement between two reviewers, a consensus or a third experienced reviewer was requested to arbitrate the result. 


\section{Methodological quality and risk of bias analysis}

A quality evaluation of the methodological soundness was performed according to the guidelines described in the Animal Research: Reporting of In Vivo Experiments (ARRIVE) guidelines. ${ }^{11}$ The seven main evaluation criteria were selected and listed in Table 1. A study was graded to have high quality with low risk of bias if it reached more than three "yes" answers to the seven criteria, moderate quality with moderate risk of bias if it reached two or three "yes" answers, and low quality with high risk of bias if it reached only one "yes" answer.

Two reviewers examined and evaluated the methods and results sections of each study independently, differences were solved by rereading and discussion until consensus was reached. The risk of bias graphic was generated by using Review Manager Software (5.1, Revman Version, Cochrane Community).

\section{Date extraction and approach to synthesis}

Studies were identified during the search process by keywords; then, the included articles were well studied by two reviewers, and the following data was collected: author, year of publication, animal type, insertion region, total number of micro-screws, size of micro-screws, loading force, observation time, failed number of micro-screws, failure rate, and the values for MRT and BIC.

A meta-analysis would be conducted if the summary effect size fitted the following criteria: low risk of bias in studies, consistent effect size across studies, low reporting bias, a high number of studies and low heterogeneity between studies. Such a qualitative synthesis would be conducted systematically by comparing the results from individual studies if the heterogeneity was high, summary effect sizes were inconsistent and risk of bias across the studies was high.

Table 1. Methodological quality and risk of bias of the selected studies with the Animal Research Reporting of In Vivo Experiments (ARRIVE) guidelines.

\begin{tabular}{|c|c|c|c|c|c|c|c|c|c|}
\hline Author, year & $\begin{array}{l}\text { Sample Size } \\
\text { Calculation }\end{array}$ & Randomization & $\begin{array}{c}\text { Blind } \\
\text { Outcome } \\
\text { Assessment }\end{array}$ & $\begin{array}{c}\text { Baseline } \\
\text { Comparability }\end{array}$ & $\begin{array}{c}\text { Animal } \\
\text { Number } \\
\text { Description }\end{array}$ & $\begin{array}{c}\text { Ethical } \\
\text { Statement }\end{array}$ & $\begin{array}{c}\text { Competing } \\
\text { Interest } \\
\text { Declaration }\end{array}$ & $Q$ & $\mathrm{R}$ \\
\hline \multicolumn{10}{|l|}{ Loading or not } \\
\hline Catharino, 2014 & No & No & No & No & Yes & Yes & Yes & M & M \\
\hline Serra, 2008 & No & No & No & No & Yes & Yes & No & M & M \\
\hline Serra, 2010 & No & No & No & No & Yes & Yes & Yes & M & M \\
\hline Chen, 2009 & No & No & No & No & Yes & No & Yes & M & M \\
\hline Mo, 2010 & No & No & No & No & Yes & Uncertain & No & L & $\mathrm{H}$ \\
\hline Goymen, 2015 & No & No & No & No & Yes & Uncertain & Yes & M & M \\
\hline \multicolumn{10}{|c|}{ Healing time before loading } \\
\hline Zhu, 2011 & No & No & No & No & Yes & No & No & L & $\mathrm{H}$ \\
\hline Shan, 2013 & No & No & No & No & Yes & No & No & L & $\mathrm{H}$ \\
\hline Yano, 2006 & No & No & No & No & Yes & Uncertain & No & L & $\mathrm{H}$ \\
\hline Zhang, 2010 & No & No & No & No & Yes & No & No & L & $\mathrm{H}$ \\
\hline Deguchi, 2003 & No & No & No & No & Yes & Yes & No & $M$ & M \\
\hline Paula, 2013 & No & No & No & No & Yes & Yes & Yes & M & M \\
\hline \multicolumn{10}{|l|}{ Loading magnitude } \\
\hline Zhang, 2008 & No & Yes & No & No & Yes & No & No & M & M \\
\hline Buchter, 2005 & No & No & No & No & Yes & Yes & No & M & M \\
\hline \multicolumn{10}{|l|}{ Loading direction } \\
\hline Park, 2011 & No & No & No & No & Yes & Yes & No & M & M \\
\hline Cho, 2010 & No & No & No & No & Yes & Uncertain & No & $\mathrm{L}$ & $\mathrm{H}$ \\
\hline
\end{tabular}




\section{Results}

\section{Study selection}

From the studies found by the keyword search, a total of 1011 records were found. After removing the duplicates, there were 458 studies left. The systematic selection by inclusion criteria and exclusion criteria resulted in 16 studies, 13 studies in English and 3 studies in Chinese. The flow of the selection process is shown in Figure 1.

\section{Quality analysis and risk of bias assessment result}

The methodological quality and the risk of bias analysis were evaluated with rigorous precision and the result is shown in Table 1 . Among the 16 studies, 10 studies were categorized as medium quality with a medium risk of bias, and no study obtained an evaluation of high quality. The remaining six studies were considered to be low quality with a high risk of bias. The percentage and summary of the differences between assessment results of the methodology of studies is shown in Figure 2 and Figure 3.

Based on the results of quality analysis and risk of bias analysis above, the data were not suitable to conduct a meta-analysis of the summary evaluation items. Therefore, a systematic review was conducted.

\section{Study characteristics and study classification}

Overall, the analyzed data was based on 102 animals and 450 micro-screws that were used. The detailed description and summary of the characteristics of the 16 included studies are given in Table 2. According to the types of loading intervention, these 16 animal studies reporting the

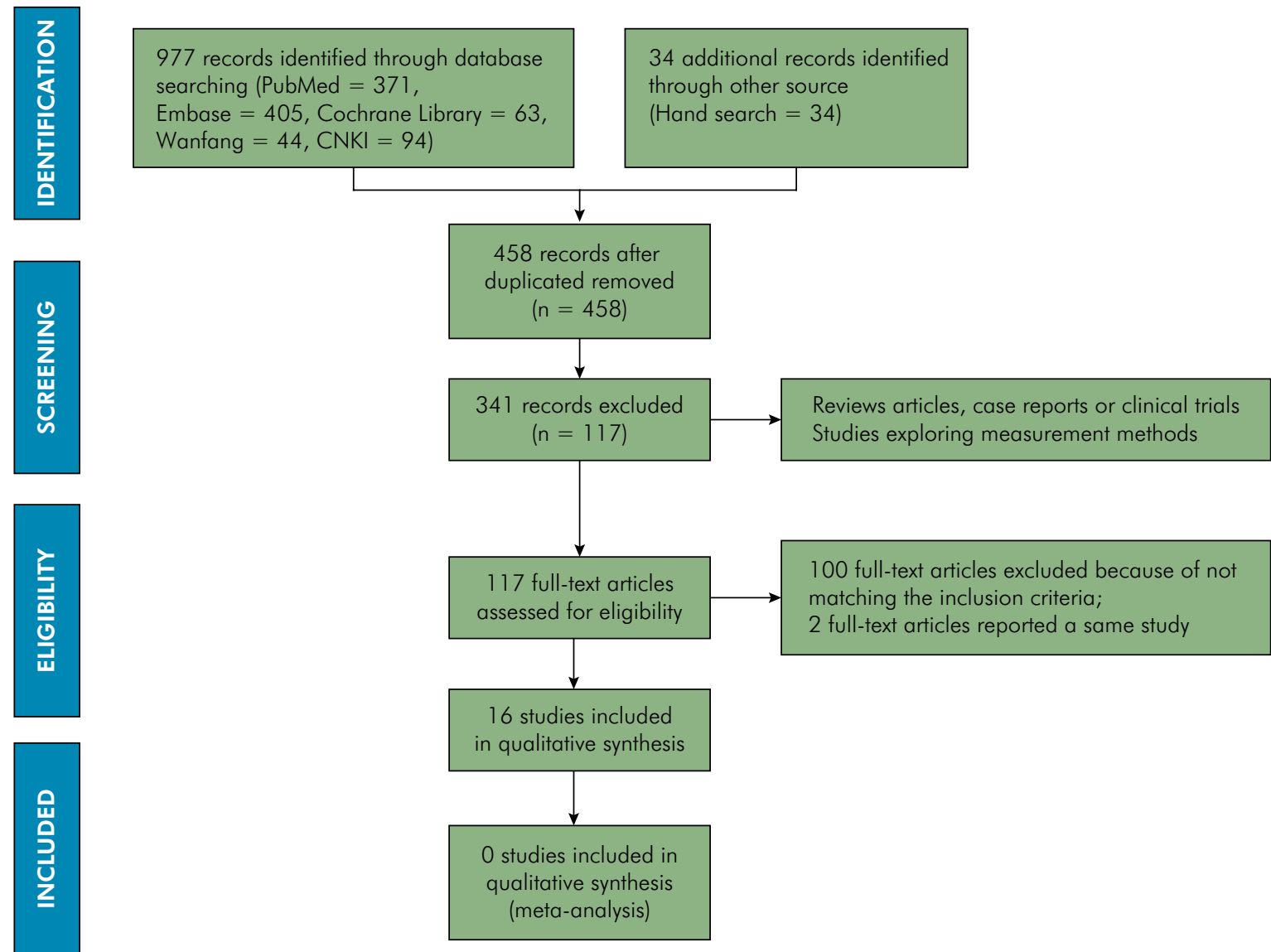

Figure 1. PRISMA flowchart of study selection. 


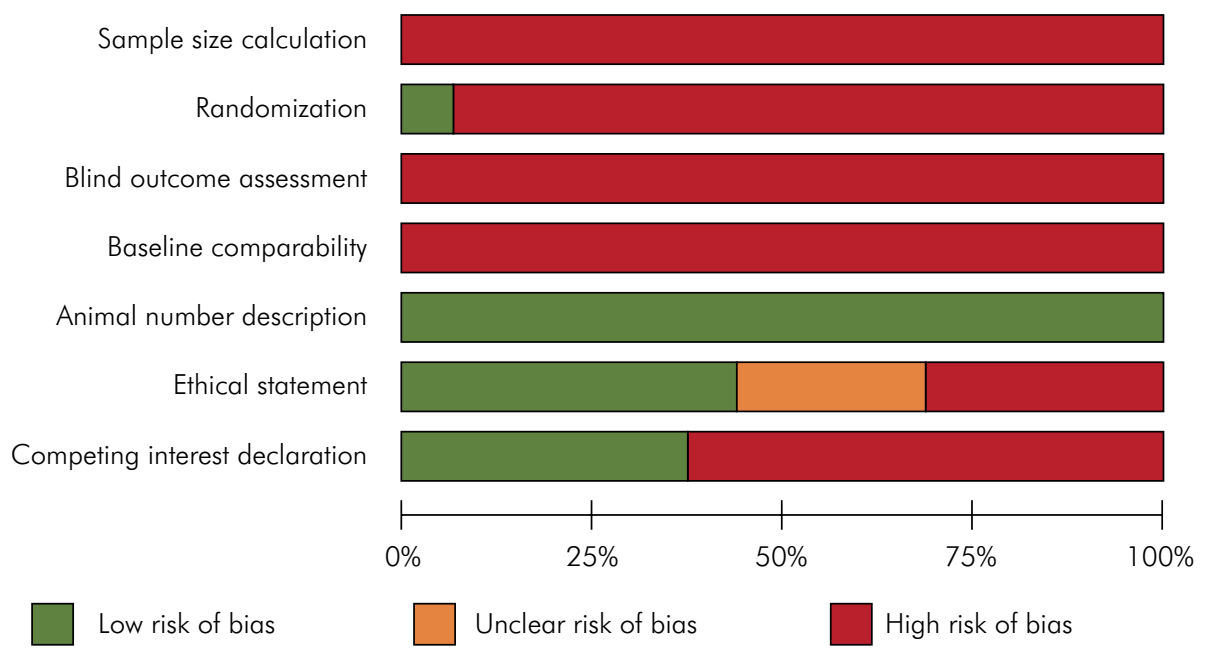

Figure 2. Judgements about each risk of bias item presented as percentages across all included studies.

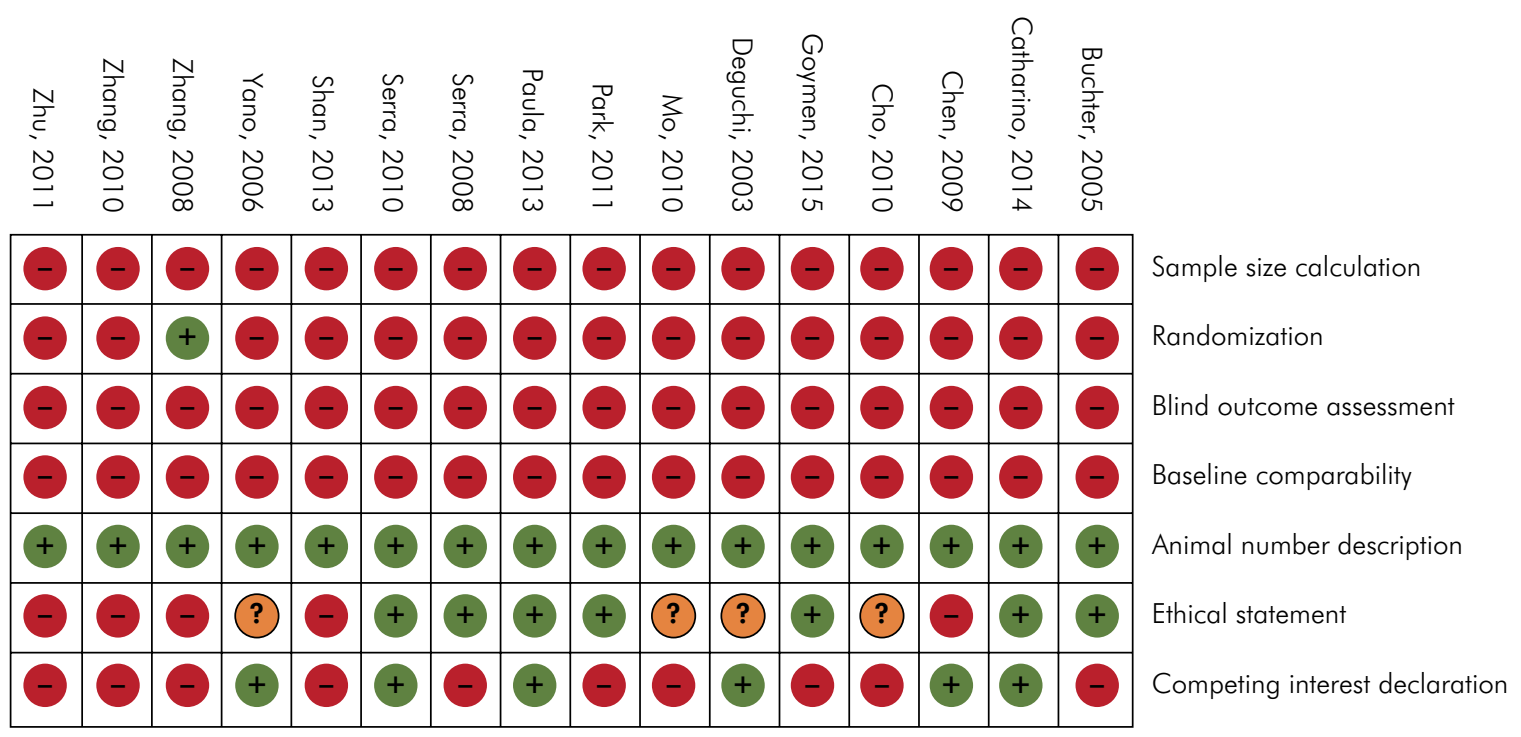

Figure 3. Risk of bias summary review of authors' judgements about each risk of bias item for each included study.

influence of variable loading conditions on the longterm stability of micro-screws could be categorized into following four groups.

\section{Group 1: Loading or not}

Six studies were classified into this group. The data collection results showed that five studies ${ }^{12,13,14,15,16}$ reported no dropping of the micro-screws in either the loading or non-loading groups, and only one study ${ }^{17}$ reported the dropping of micro-screws; the failure rate of the loading group was lower than the non-loading group. As for MRT values, one study reported lower values in the loading group compared to the non-loading group $(\mathrm{p}<0.05)$. However, in another study with more samples per group ${ }^{13}$, the difference in MRT values between the loading group and the non-loading group was not significant $(p>0.05)$. Three studies ${ }^{14,15,17}$ reported that there was no statistical difference in BIC values between the loading group and the non-loading group $(p>0.05)$, while only one study ${ }^{16}$ reported that the BIC values in the loading group were higher than those in the non-loading group $(\mathrm{p}<0.05)$. 


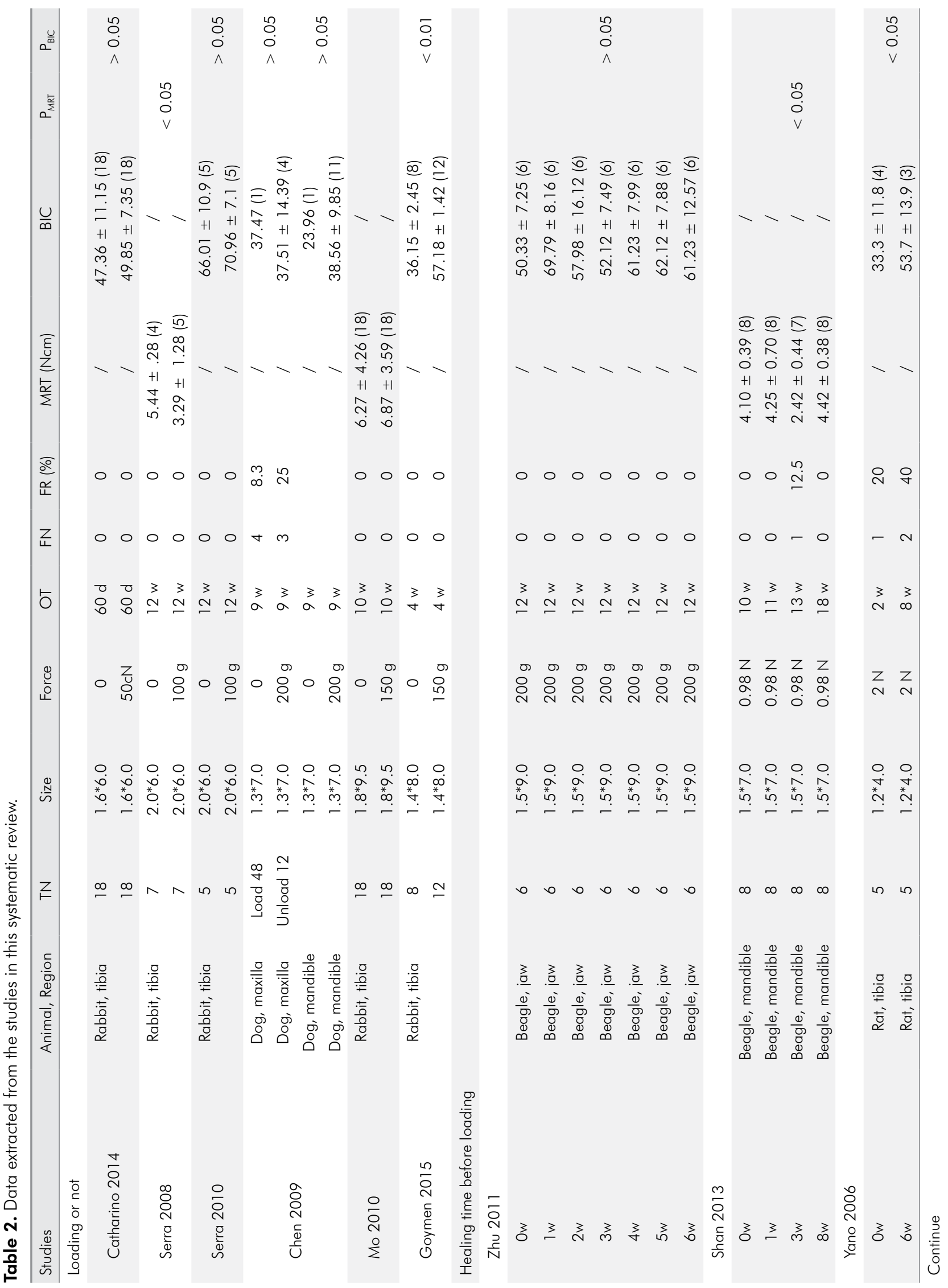




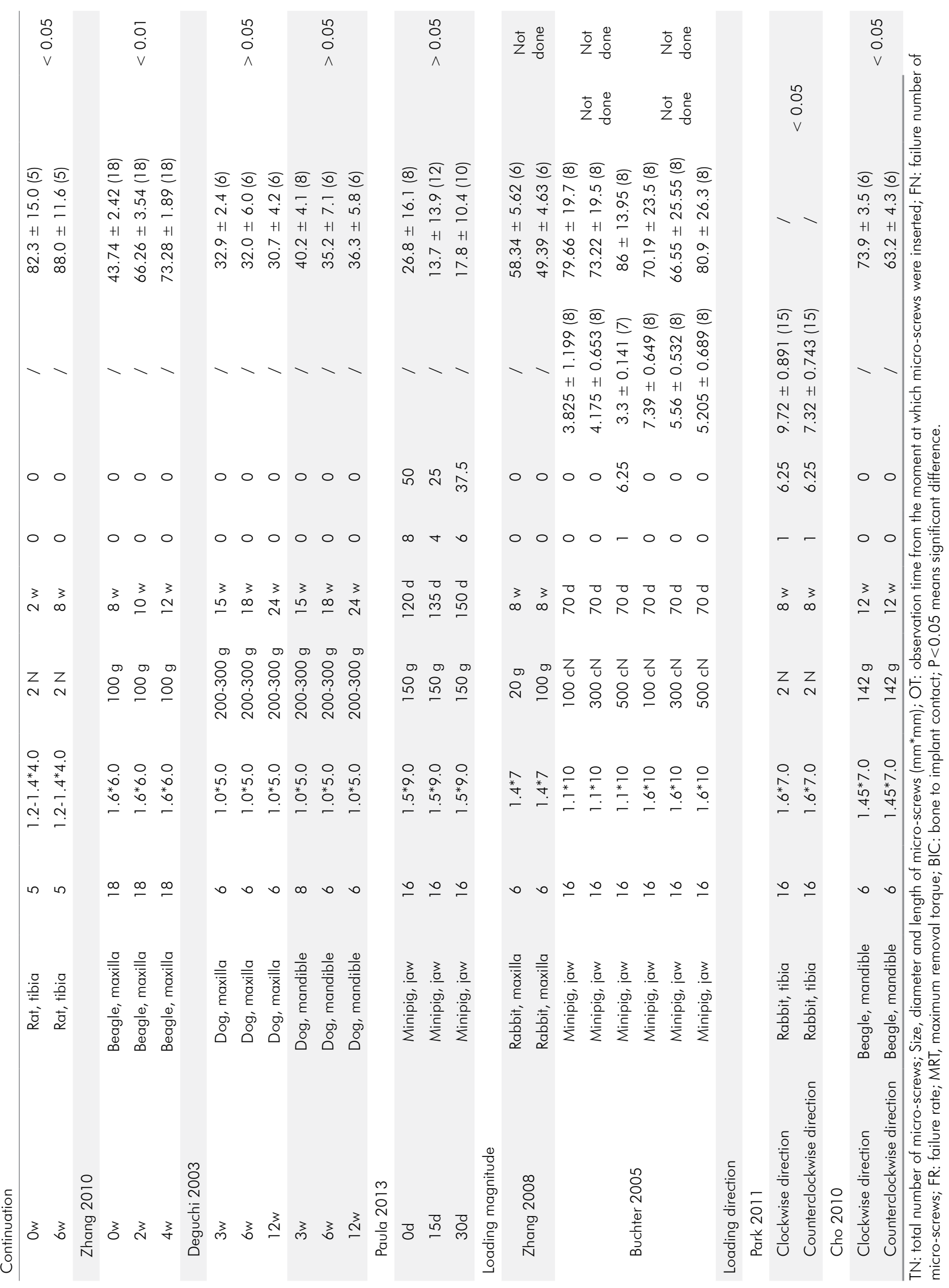




\section{Group 2: Healing time before loading}

Six studies were classified into this group. There were three studies ${ }^{18,19,20}$ that did not report the dropping of micro-screws in the evaluated healing periods. One study ${ }^{21}$ reported a failure of one micro-screw in the 3-week healing time group, and it also showed that the MRT values in the 3-week healing time group were significantly lower than other groups $(p<0.05)$. As for BIC values, one study ${ }^{22}$ reported a higher value in the 6-week healing time group compared with the immediate loading group $(p<0.05)$. Another study ${ }^{19}$ showed that the BIC values in the 4-week healing time group were higher than those in the 2-week healing time group ( $\mathrm{p}<0.05$ ), and the BIC values in the 2-week healing time group were also higher than those in the immediate loading group $(\mathrm{p}<0.05)$. However, three studies ${ }^{18,20,23}$ reported that the difference of BIC values between various healing time groups was not significant ( $p>0.05)$.

\section{Group 3: Loading magnitude}

Two studies were classified into this group. One study ${ }^{24}$ without statistical analysis results reported the drop of one micro-screw under a high-intensity load of $500 \mathrm{cN}$, and the MRT values in the $500 \mathrm{cN}$ force group were lower than both the $100 \mathrm{cN}$ force and $300 \mathrm{cN}$ force groups. However, the $500 \mathrm{cN}$ force group had a higher BIC value than both the $100 \mathrm{cN}$ and $300 \mathrm{cN}$ force groups. The other study, ${ }^{25}$ also without a statistically significant $\mathrm{p}$-value, reported that the BIC values in the $100 \mathrm{~g}$ force group were lower than those in the $200 \mathrm{~g}$ force group.

\section{Group 4: Loading direction}

There were two studies comparing the effect of clockwise and counterclockwise load on the long-term stability of micro-screws. One study ${ }^{26}$ calculated the MRT value, and the result showed that the value in the counterclockwise load group was lower than that in the clockwise load group with a statistically significant difference $(p<0.05)$. The other study ${ }^{27}$ calculated the BIC value, and the outcome suggested that the value in the counterclockwise load group was also lower than that in the clockwise load group $(\mathrm{p}<0.05)$.

\section{Discussion}

The selection of several articles of a single study could mislead the real effects of an intervention. ${ }^{28}$ In this systematic review, two articles ${ }^{24,29}$ based on the same study were published by a single author. The author separated the experimental result into two parts according to the evaluation items and published two papers-one on the biomechanical outcome and the other on the histological outcome. During the study inclusion process, we combined these two articles into one study.

A systematic review's cornerstone is based on the assessment of risk of bias, and it can be used as a framework to explain conflicting outcomes. The risk of bias evaluation in this systematic review followed the ARRIVE guidelines. To our knowledge, there are no specific guidelines existing for an in vivo database. In 2010, the ARRIVE guidelines were published to address the growing concerns with poor experiment design and lack of transparent reporting of in vivo experiments ${ }^{11}$. The guidelines consist of a checklist of 20 items, and we took seven main criteria for the evaluation of methodological quality and risk of bias analysis.

Although the outcome evaluation items of the selected studies were quantitative data, the data together were not suitable to conduct a quantitative synthesis, as the heterogeneity between the studies in each group was high. This heterogeneity could be caused by the following factors: differences in animal models and insertion into bone tissues; differences in the diameter and length of micro-screws; differences in the magnitude of load; differences in observation time for long-term stability evaluation and differences in the calculation number of samples. Therefore, a systematic review was conducted.

\section{Loading or not}

Some researchers have investigated the effect of loading force on the bone healing process and whether loading is disadvantageous to the long-term stability of micro-screws has been under discussion. In summary of the qualitative data analysis results, 5 of the 6 studies reported that loading would not destroy the long-term stability of micro-screws, and 
the difference between the loading group and the non-loading group was not significant.

Regardless of immediate loading, Serra ${ }^{12}$ found no statistical difference between the loading and the non-loading group when evaluating the MRT and BIC values. Moreover, loading did not affect the amount of osseointegration in the tensional and compressional areas, and there was no statistical difference between two areas. Later, Serra ${ }^{15}$ designed another study to evaluate the interface reactions at different stages of osseointegration around micro-screws that were immediately loaded with $1 \mathrm{~N}$. After 1-week and 4-week healing times, they found that there was no histologic difference in $\mathrm{BIC}$ and BA values between the loading group and the non-loading group, which suggested that the formation of native lamellar bone would not be impaired by immediate loading. After a 12-week healing duration, the bone deposition rate was greater in the loading group, indicating that proper loading may improve the extent of final osseointegration and the long-term stability of micro-screws. This finding was consistent with the previous research result reported by Luzi, ${ }^{30}$ who presented an investigation to evaluate the reaction of bone tissue to immediate loading $(50 \mathrm{cN})$ of micro-screws.

As biomechanical and histological evaluation items can be influenced by many factors, additional concern should be given to the loading protocols and animal species when comparing different relative reports. Recently, Catharino ${ }^{14}$ quantified the process of bone healing around micro-screws during four different time periods, with or without immediate loading. They found that the histomorphometric values for BIC increased significantly throughout the healing period, regardless of whether a load was applied. They suggested that the loading of a light, immediate and continuous force $(50 \mathrm{cN})$ would not negatively affect the process of new bone formation; rather, it activated the process of tissue adaption and the remodeling of surrounding bone.

Although micro-screws can withstand loading forces of 250-300 g during the treatment period, many scholars still emphasize that an immediate load greater than $100 \mathrm{~g}$ will cause a higher incidence of bone fractures and loss of micro-screws in the long run. ${ }^{14,15,30}$ In order to reduce marginal bone fracture and obtain long-term stability with loading, a low-intensity load below $100 \mathrm{~g}$ force and predrilling of cortical bone before inserting micro-screws were advised.

\section{Healing time before loading}

To shorten orthodontic treatment time, immediate or early loading of micro-screws after insertion is desired. Is immediate loading beneficial to osseointegration in the long-term period? How long should we wait before loading to get sufficient long-term stability? Melsen ${ }^{31}$ observed the bone-implant contact area that was under early loading and found that the interface was lacking intimate bone contact because of the interplay of fibrous tissue. However, Aldikacti ${ }^{32}$ suggested that after 6-week healing time, micro-screws obtained enough biological fixation and were able to resist a load. Recently, some scholars ${ }^{30,33}$ presented that 1 or 2 weeks of healing time before loading was enough for micro-screws to become stable. As we can see, the published papers have not produced an united answer. In summary of the qualitative data, $3^{18,20,23}$ of the 6 studies reported that the various healing periods tested before loading had no influence on the long-term stability of micro-screws, and low-intensity immediate loading or a minimum of 3 weeks of healing time was acceptable. Two studies ${ }^{19,22}$ indicated that a period of healing time before loading was beneficial for the long-term stability of microscrews. The last ${ }^{21}$ study reported that loading after 3-week healing was disadvantageous to the stability of micro-screws.

$Z_{\text {hao }}{ }^{34}$ designed an animal experimentation and found that both osseointegration and peri-implant trabecular bone density in the immediately loaded and 1-week healing time groups were significantly lower than that in the 3-, 5-, and 7-week healing time groups, and there were no obvious differences between the $3-, 5-$, and 7-week groups. They concluded that after 3 weeks of healing time, the bone-screw fixture was strong enough to support loading. This was supported by another study reported by Zhang, ${ }^{19}$ which used micro CT to provide comprehensive observation of the bone-screw surface in three dimensions. Their 
result showed that there was a significant rising tendency of micro-screws' long-term stability as healing time went by, and after 3-week healing time, the integration of micro-screws was strong enough for clinical use. Therefore, early loading after a 3-week healing duration may have a positive impact on the long-term stability of micro-screws.

However, some scholars came up with different opinions. Paula ${ }^{23}$ designed three loading time points (immediately, after 15 days and after 30 days) to evaluate the effect of healing time on micro-screws' stability. Regarding the values of BIC and BA, there was no statistical difference among the three groups. The author confirmed that early loading would not affect micro-screws' stability, and this could be supported by other studies. ${ }^{15,35,36}$ Recently, Ramazanzadeh ${ }^{37}$ designed an animal study and found that at 8 weeks, BIC values of the immediately loaded group were slightly less than the 4-week healing time group, and the differences were not statistically significant. It confirmed that healing time had no obvious effect on micro-screw's stability.

From the above discussion, it is possible to apply loading immediately after insertion, but this may differ depending on the species of animal, the quality of surrounding bone, the types of micro-screws and the loading magnitude. Therefore, for safety, a minimum healing time of 3 weeks was suggested for micro-screws' long-term stability.

\section{Loading magnitude}

Micro-screws have mainly been used as orthodontic anchorage systems to withstand load. Although the powerful function of micro-screws in clinical application has been confirmed, less is known about their long-term stability with respect to loading magnitudes. The loading magnitude applied to micro-screws has always been a controversial issue, just as the inconsistent qualitative data from the two selected studies showed.

Buchter ${ }^{24}$ gained insight into the optimized loading magnitudes of micro-screws and found that as long as loads did not exceed a tolerable strain level, the loading would not impede the process of peri-implant bone healing. They suggested that by controlling loading magnitude under a threshold of
$300 \mathrm{cN}$, micro-screws can be loaded immediately and obtain a high mean removal torque without reducing the micro-screws' long-term stability. As relative studies continued, most of the scholars ${ }^{38,39,40}$ indicated that micro-screws would loosen gradually and face dropping when the loading magnitude reached above $200 \mathrm{~g}$, and the suggested magnitude was between $100 \mathrm{~g}$ and $200 \mathrm{~g}$.

In conclusion, the relative studies above indicate that a moderate loading magnitude of $100-200 \mathrm{~g}$ will not impact the long-term stability of micro-screws. But once we have confirmed the exact kind of loading magnitude that is beneficial to the long-term stability of micro-screws, we should also investigate whether this force will compromise the effect of tooth movement or increase the risk of root resorption.

\section{Loading direction}

Most micro-screw studies estimated only simple lateral forces on the long-term stability of micro-screws, and few studies have reported the effect of loading direction on the stability of micro-screws. In summary of the qualitative data, two studies reported a consistent conclusion that counterclockwise loading compared with clockwise loading could decrease the long-term stability of micro-screws.

$\mathrm{Cho}^{27}$ studied the effect of loading direction on the long-term stability of micro-screws. They found that the BIC values in the counterclockwise group were much lower than those in the clockwise group at 12 weeks after insertion. Therefore, counterclockwise loading was considered the reason for loosening of micro-screws and poor long-term stability. Later, Park ${ }^{26}$ did a more detailed study in rabbit tibias and found that the removal torque values were similar in the lateral, clockwise and counterclockwise groups after 1 week of healing. After 8 weeks of healing, the counterclockwise group had a larger bone defect area than the control and clockwise groups. This may be the reason that counterclockwise rotational movement loads more pressure on the bone surface.

Although extensive studies have not yet been performed, it is accepted in clinical application that loading with counterclockwise rotational movement may be a risk factor for the long-term stability of 
micro-screws. Thus, counterclockwise rotational movement of a large magnitude should be avoided. ${ }^{13}$ As counterclockwise loading is in the direction of loosening micro-screws, we suggest that a large magnitude of force or continuous force loading should be delayed.

As discussed above, immediate loading below $100 \mathrm{~g}$ force, more than 3-week healing time, regular loading under $200 \mathrm{~g}$ force and a clockwise direction of force contributed to the long-term stability of microscrews. However, most of the existing studies only evaluated the effect of one loading condition factor on the long-term stability of micro-screws. Additional studies pertinent to the combination of loading magnitudes, loading periods and loading directions will be needed to determine which combined loading condition is better for micro-screws to obtain sufficient long-term stability.

\section{Conclusion}

In this systematic review, the failure rate, the biomechanical item of MRT values and the histomorphometric item of BIC values were analyzed to evaluate the long-term stability of micro-screws, and the following results were obtained:

a. The presence of an immediate load below $100 \mathrm{~g}$ force will not inhibit the osseointegration process or affect the long-term stability of micro-screws.

b. Although micro-screws' long-term stability will not be influenced significantly by various healing periods before loading, it is still emphasized that micro-screws can be strong enough to support loading after a minimum healing time of 3 weeks.

c. A moderate loading magnitude of $100-200 \mathrm{~g}$ will not impede the process of bone remodeling and has no obvious effect on the long-term stability of micro-screws.

d. Compared with clockwise loading, counterclockwise loading may be a risk factor for reducing the long-term stability of micro-screws.

\section{Acknowledgments}

The authors certify that they have no commercial or associative interest that represents a conflict of interest in connection with the manuscript. This work was supported by the National Natural Science Foundation of China [grant number 815008131003912].

\section{References}

1. Sfondrini MF, Gandini P, Alcozer R, Vallittu PK, Scribante A. Failure load and stress analysis of orthodontic miniscrews with different transmucosal collar diameter. J Mech Behav Biomed Mater. 2018 Nov;87:132-7. https://doi.org/10.1016/i.jmbbm.2018.07.032

2. Pimentel AC, Manzi MR, Prado Barbosa AJ, Cotrim-Ferreira FA, Guedes Carvalho PE, de Lima GF, et al. Mini-implant screws for bone-borne anchorage: a biomechanical in vitro study comparing three diameters. Int J Oral Maxillofac Implants. 2016 Sep-Oct;31(5):1072-6. https://doi.org/10.11607/jomi.4390

3. Arantes VD, Corrêa CB, Lunardi N, Boeck Neto RJ, Spin-Neto R, Boeck EM. Insertion angle of orthodontic mini-implants and their biomechanical performance: finite element analysis. Rev Odontol UNESP. 2015 Oct;44(5):273-9. https://doi.org/10.1590/1807-2577.0081

4. Maino BG, Di Blasio A, Spadoni D, Ravanetti F, Galli C, Cacchioli A, et al. The integration of orthodontic miniscrews under mechanical loading: a pre-clinical study in rabbit. Eur J Orthod. 2017 Oct;39(5):519-27. https://doi.org/10.1093/ejo/cjw069

5. Yao CC, Chang HH, Chang JZ, Lai HH, Lu SC, Chen YJ. Revisiting the stability of mini-implants used for orthodontic anchorage. J Formos Med Assoc. 2015 Nov;114(11):1122-8. https://doi.org/10.1016/i.jfma.2014.08.001

6. Quraishi E, Sherriff M, Bister D. Peak insertion torque values of five mini-implant systems under different insertion loads. J Orthod. 2014 Jun;41(2):102-9. https://doi.org/10.1179/1465313313Y.0000000084

7. Jang TH, Park JH, Moon W, Chae JM, Chang NY, Kang KH. Effects of acid etching and calcium chloride immersion on removal torque and bone-cutting ability of orthodontic mini-implants. Am J Orthod Dentofacial Orthop. 2018 Jul;154(1):108-14. https://doi.org/10.1016/i.ajodo.2017.10.032

8. Chatzigianni A, Keilig L, Duschner H, Götz H, Eliades T, Bourauel C. Comparative analysis of numerical and experimental data of orthodontic mini-implants. Eur J Orthod. 2011 Oct;33(5):468-75. https://doi.org/10.1093/ejo/cir097

9. Nienkemper M, Handschel J, Drescher D. Systematic review of mini-implant displacement under orthodontic loading. Int J Oral Sci. 2014 Mar;6(1):1-6. https://doi.org/10.1038/ijos.2013.92 
Evaluation of the long-term stability of micro-screws under different loading protocols: a systematic review

10. Higgins JP, Green S. Cochrane handbook for systematic reviews of interventions, version 5.1.0 (updated March 2011). London: Cochrane Collaboration; 2011.

11. Kilkenny C, Browne WJ, Cuthill IC, Emerson M, Altman DG. Improving bioscience research reporting: the ARRIVE guidelines for reporting animal research. Osteoarthritis Cartilage. 2012 Apr;20(4):256-60. https://doi.org/10.1016/i.joca.2012.02.010

12. Serra G, Morais LS, Elias CN, Meyers MA, Andrade L, Muller C, et al. Sequential bone healing of immediately loaded mini-implants. Am J Orthod Dentofacial Orthop. 2008 Jul;134(1):44-52. https://doi.org/10.1016/i.ajodo.2006.09.057

13. Mo SS, Kim SH, Kook YA, Jeong DM, Chung KR, Nelson G. Resistance to immediate orthodontic loading of surface-treated mini-implants. Angle Orthod. 2010 Jan;80(1):123-9. https://doi.org/10.2319/030309-123.1

14. Catharino PC, Dominguez GC, Pinto DS Jr, Morea C. Histologic, histomorphometric, and radiographic monitoring of bone healing around in-office-sterilized orthodontic mini-implants with or without immediate load: study in rabbit tibiae. Int J Oral Maxillofac Implants. 2014 Mar-Apr;29(2):321-30. https://doi.org/10.11607/jomi.2842

15. Serra G, Morais LS, Elias CN, Meyers MA, Andrade L, Müller CA, et al. Sequential bone healing of immediately loaded mini-implants: histomorphometric and fluorescence analysis. Am J Orthod Dentofacial Orthop. 2010 Jan;137(1):80-90. https://doi.org/10.1016/i.ajodo.2007.12.035

16. Goymen M, Isman E, Taner L, Kurkcu M. Histomorphometric evaluation of the effects of various diode lasers and force levels on orthodontic mini screw stability. Photomed Laser Surg. 2015 Jan;33(1):29-34. https://doi.org/10.1089/pho.2014.3833

17. Chen Y, Kang ST, Bae SM, Kyung HM. Clinical and histologic analysis of the stability of microimplants with immediate orthodontic loading in dogs. Am J Orthod Dentofacial Orthop. 2009 Aug;136(2):260-7. https://doi.org/10.1016/i.ajodo.2007.10.046

18. Zhu LY, Yan YQ, Gao YM. [A study on the stability of miniscrew on different loading time as orthodontic anchorage]. Shanghai Kou Qiang Yi Xue. 2011 Dec;20(6):590-4. Chinese.

19. Zhang L, Zhao Z, Li Y, Wu J, Zheng L, Tang T. Osseointegration of orthodontic micro-screws after immediate and early loading. Angle Orthod. 2010 Mar;80(2):354-60. https://doi.org/10.2319/021909-106.1

20. Deguchi T, Takano-Yamamoto T, Kanomi R, Hartsfield JK Jr, Roberts WE, Garetto LP. The use of small titanium screws for orthodontic anchorage. J Dent Res. 2003 May;82(5):377-81. https://doi.org/10.1177/154405910308200510

21. Shan L, Zhou G, Li X, Qie H, Dong F. [Mini-implant stability analysis at different healing times before loading]. Hua Xi Kou Qiang Yi Xue Za Zhi. 2013 Dec;31(6):557-60. Chinese.

22. Yano S, Motoyoshi M, Uemura M, Ono A, Shimizu N. Tapered orthodontic miniscrews induce bone-screw cohesion following immediate loading. Eur J Orthod. 2006 Dec;28(6):541-6. https://doi.org/10.1093/ejo/cjl044

23. Oltramari-Navarro PV, Navarro RL, Henriques JF, Cestari TM, Francischone CE, Taga R, et al. The impact of healing time before loading on orthodontic mini-implant stability: a histomorphometric study in minipigs. Arch Oral Biol. 2013 Jul;58(7):806-12. https://doi.org/10.1016/j.archoralbio.2012.12.010

24. Büchter A, Wiechmann D, Koerdt S, Wiesmann HP, Piffko J, Meyer U. Load-related implant reaction of mini-implants used for orthodontic anchorage. Clin Oral Implants Res. 2005 Aug;16(4):473-9. https://doi.org/10.1111/j.1600-0501.2005.01149.x

25. Zhang XY, Lv Y, Wang BK. [Histological research on immediate-loading miniscrew as orthodontic anchorage]. J Orthod. 2008;15(3):118-20. Chinese

26. Park KH, Lee EM, Shin SI, Kim SH, Park YG, Kim SJ. Evaluation of the effect of force direction on stationary anchorage success of mini-implant with a lever-arm-shaped upper structure. Angle Orthod. 2011 Sep;81(5):776-82. https://doi.org/10.2319/092810-566.1

27. Cho YM, Cha JY, Hwang CJ. The effect of rotation moment on the stability of immediately loaded orthodontic miniscrews: a pilot study. Eur J Orthod. 2010 Dec;32(6):614-9. https://doi.org/10.1093/ejo/cjq008

28. Higgins JP, Green S, eds. Cochrane handbook for systematic reviews of interventions. Chichester, United Kingdom: John Wiley \& Sons; 2008. https://doi.org/10.1002/9780470712184

29. Büchter A, Wiechmann D, Gaertner C, Hendrik M, Vogeler M, Wiesmann HP, et al. Load-related bone modelling at the interface of orthodontic micro-implants. Clin Oral Implants Res. 2006 Dec;17(6):714-22. https://doi.org/10.1111/j.1600-0501.2006.01233.x

30. Luzi C, Verna C, Melsen B. Immediate loading of orthodontic mini-implants: a histomorphometric evaluation of tissue reaction. Eur J Orthod. 2009 Feb;31(1):21-9. https://doi.org/10.1093/ejo/cjn087

31. Melsen B, Costa A. Immediate loading of implants used for orthodontic anchorage. Clin Orthod Res. 2000 Feb;3(1):23-8. https://doi.org/10.1034/j.1600-0544.2000.030105.x

32. Aldikaçti M, Açikgöz G, Türk T, Trisi P. Long-term evaluation of sandblasted and acid-etched implants used as orthodontic anchors in dogs. Am J Orthod Dentofacial Orthop. 2004 Feb;125(2):139-47. https://doi.org/10.1016/S0889-5406(03)00629-2

33. Kim JW, Ahn SJ, Chang YI. Histomorphometric and mechanical analyses of the drill-free screw as orthodontic anchorage. Am J Orthod Dentofacial Orthop. 2005 Aug;128(2):190-4. https://doi.org/10.1016/i.ajodo.2004.01.030

34. Zhao L, Xu Z, Yang Z, Wei X, Tang T, Zhao Z. Orthodontic mini-implant stability in different healing times before loading: a microscopic computerized tomographic and biomechanical analysis. Oral Surg Oral Med Oral Pathol Oral Radiol Endod. 2009 Aug;108(2):196-202. https://doi.org/10.1016/i.tripleo.2009.03.023 
35. Crismani AG, Bertl MH, Celar AG, Bantleon HP, Burstone CJ. Miniscrews in orthodontic treatment: review and analysis of published clinical trials. Am J Orthod Dentofacial Orthop. 2010 Jan;137(1):108-13. https://doi.org/10.1016/i.ajodo.2008.01.027

36. Woods PW, Buschang PH, Owens SE, Rossouw PE, Opperman LA. The effect of force, timing, and location on bone-to-implant contact of miniscrew implants. Eur J Orthod. 2009 Jun;31(3):232-40. https://doi.org/10.1093/ejo/cjn091

37. Ramazanzadeh BA, Fatemi K, Dehghani M, Mohtasham N, Jahanbin A, Sadeghian H. Effect of healing time on bone-implant contact of orthodontic micro-implants: a histologic study. ISRN Dent. 2014 Apr;2014(1):179037. https://doi.org/10.1155/2014/179037

38. Chen Y, Kyung HM, Zhao WT, Yu WJ. Critical factors for the success of orthodontic mini-implants: a systematic review. Am J Orthod Dentofacial Orthop. 2009 Mar;135(3):284-91. https://doi.org/10.1016/i.ajodo.2007.08.017

39. Motoyoshi M, Yano S, Tsuruoka T, Shimizu N. Biomechanical effect of abutment on stability of orthodontic mini-implant. A finite element analysis. Clin Oral Implants Res. 2005 Aug;16(4):480-5. https://doi.org/10.1111/j.1600-0501.2005.01130.x

40. Chaddad K, Ferreira AF, Geurs N, Reddy MS. Influence of surface characteristics on survival rates of mini-implants. Angle Orthod. 2008 Jan;78(1):107-13. https://doi.org/10.2319/100206-401.1 